\title{
Cooperative Spectrum Sensing over Non-Identical Nakagami Fading Channels
}

\author{
Anlei Rao and Mohamed-Slim Alouini \\ Electrical Engineering Program \\ Division of Physical Sciences and Engineering \\ King Abdullah University of Science and Technology (KAUST) \\ Thuwal, Mekkah Province, Saudi Arabia \\ E-mail:\{anlei.rao; mohamed.alouini\}@kaust.edu.sa
}

\begin{abstract}
Previous works in cooperative spectrum sensing assumed that the channels for sensing and reporting are independent identical distributed (i.i.d). A more practical and appropriate assumption, however, should be that the sensing channels and reporting channels are independent but not necessarily identically distributed (i.n.i.d). In this paper, we derive the falsealarm probability and the detection probability of cooperative spectrum sensing with energy fusion over i.n.i.d Nakagami fading channels. Selected numerical results show that cooperative spectrum sensing still gives considerably better performance results even over i.n.i.d fading channels.

Index Terms-Cooperative spectrum sensing; Nakagami fading; Energy detection;
\end{abstract}

\section{INTRODUCTION}

The dramatic growth of wireless services over the last decade explains the great demand for radio spectrum. The spectrum resource, however, is limited and most of it has already been allocated. On the other hand, many portions of the allocated spectrum are not fully utilized. In order to deal with this conflict between spectrum scarcity and spectrum under-utilization, cognitive radio has been proposed as a revolutionary technology for the next generation of wireless communication networks [1].

To guarantee that the operation of the primary users is not affected, the secondary users must possess the ability of sensing the presence of active primary users, and this process is known as spectrum sensing. In some specific environment, multi-path fading and shadowing may cause the disability of secondary users to detect a primary user. In order to solve such problems, multiple secondary users can cooperate with each other to conduct the spectrum sensing to achieve an improved performance [2].

Recently, cooperative sensing has been widely studied [3][4], and these studies suppose that the sensing channels are identically distributed. But this is not always the case in practice. Indeed in this case, the received signals by cooperating secondary users may experience a variety of path loss, shadowing or fading conditions. As such, a more

*This work was supported by King Abdullah University of Science and Technology (KAUST). appropriate and practical assumption is to consider i.n.i.d channels. In this paper, we consider cooperative sensing in such kind of environment and we assume that the reporting channels to the fusion center are error-free. In particular, instead of sending the binary local decision, each secondary user is assumed to transmit the normalized energy statistic to the fusion center, which makes a final decision by fusing these local energy statistics. In this context, we calculate the false-alarm probability and miss-detection probability with a threshold determined by the Neyman-Pearson criterion.

The rest of this paper is organized as follows: In Section II, the system model is introduced. Next, Section III presents the performance analysis over i.n.i.d Nakagami fading channels. Then the following section shows some selected numerical results. Finally, conclusions will be presented in Section V.

\section{SySTEM MODEL}

We consider a cooperative cognitive radio network with $N$ secondary users and a fusion center, and assume that each secondary user performs local energy sensing independently and report these energy values to the fusion center over errorfree reporting channels. We also assume that the sensing channels are independent. Different from previous works, however, these channels may be not identically distributed but subject to the same kind of fading.

With the i.n.i.d assumption made above, the signal-to-noise ratio (SNR) of the received signal from the primary user for $n$-th secondary user can be denoted as $\gamma_{n}$, with its mean $\bar{\gamma}_{n}$ and probability density function (PDF) $f_{\gamma_{n}}\left(\gamma_{n}\right)$. The $n$ th secondary user measures the normalized energy statistic of the signal it has received as $E_{n}$, which has a central chi-square distribution under the hypothesis $H_{0}$ (inactive primary user) and non-central chi-square distribution with a center parameter $2 \gamma_{n}$ under the hypothesis $H_{1}$ (active primary user) [5]:

$$
E_{n} \sim \begin{cases}\chi_{2 u}^{2} & H_{0} \\ \chi_{2 u}^{2}\left(2 \gamma_{n}\right) & H_{1},\end{cases}
$$

where $u=T W$ is the time bandwidth product with $T$ the observation time interval and $W$ the one-sided bandwidth. 
The fusion center collects the normalized energy statistic from each secondary user using the energy fusion scheme [3] to make a decision. Under perfect reporting channel conditions, the fusion energy statistic yields

$$
E=\sum_{n=1}^{N} E_{n}
$$

Based on (2), it is not difficult to show that the fusion energy statistic $E$ is also subject to a central chi-square distribution under the hypothesis $H_{0}$ and a non-central chisquare distribution with a center parameter $2 \gamma$ under the hypothesis $H_{1}$ :

$$
E \sim \begin{cases}\chi_{2 N u}^{2} & H_{0} \\ \chi_{2 N u}^{2}(2 \gamma) & H_{1}\end{cases}
$$

where $\gamma=\sum_{n=1}^{N} \gamma_{n}$ is the combined SNR with a PDF $f_{\gamma}(\gamma)$. As such, the PDF of $E$ conditioned on $\gamma$ is given by

$$
f_{E}(x)= \begin{cases}\frac{1}{2^{N u} \Gamma(N u)} x^{N u-1} \mathrm{e}^{-\frac{x}{2}} & H_{0} \\ \frac{1}{2}\left(\frac{x}{2 \gamma}\right)^{\frac{N u-1}{2}} \mathrm{e}^{-\frac{2 \gamma+x}{2}} I_{N u-1}(\sqrt{2 \gamma x}) & H_{1},\end{cases}
$$

where $\Gamma(\cdot)$ is the gamma function and $I_{M}(\cdot)$ is the $M$-th order modified Bessel function of the first kind.

To make a decision, the fusion center compares the energy statistic $E$ to a threshold $\lambda$, and decide on $H_{0}$ when $E<\lambda$ and on $H_{1}$ when $E>\lambda$. In this conditions, the false-alarm and detection probability conditioned on $\gamma$ are given by [5]

$$
\begin{aligned}
P_{f} & =\frac{\Gamma(N u, \lambda / 2)}{\Gamma(N u)} \\
P_{d} & =Q_{N u}(\sqrt{2 \gamma}, \sqrt{\lambda})
\end{aligned}
$$

where $\Gamma(\cdot, \cdot)$ is the upper incomplete gamma function, and $Q_{M}(\cdot, \cdot)$ is the generalized Marcum Q-function. The averaged detection probability can then be computed in different fading environments as

$$
\bar{P}_{d}=\int_{0}^{+\infty} Q_{N u}(\sqrt{2 \gamma}, \sqrt{\lambda}) f_{\gamma}(\gamma) d \gamma
$$

since the conditional false-alarm probability is independent of $\gamma$, then $\bar{P}_{f}=P_{f}$.

The threshold $\lambda$ can be determined using the NeymanPearson criterion. In particular, given a desired false-alarm probability $P_{f}=\alpha$, the threshold $\lambda$ can be determined and can be used in (5) to find the average detection probability.

\section{Performance Analysis}

Nakagami fading occurs for multipath scattering with relatively large delay-time spreads, with different clusters of reflected waves. It represents a wide range of multipath channels via the fading parameter $m$ [6]. For instance, the Nakagami fading includes the one-sided Gaussian fading $(m=1 / 2$, which corresponds to worst-case fading) and the Rayleigh fading $(m=1)$ as special cases. In addition, when $m>1$, a one-to-one mapping between the Rician factor and the Nakagami fading parameter allows the Nakagami fading to closely approximate the Rice fading [6]. Finally, and perhaps most importantly, the Nakagami fading often gives the best fit to urban [7] and indoor [8] multipath propagation.

For Nakagami fading channels, the local SNR of secondary users is subject to a Gamma distribution given by

$$
\begin{array}{r}
f\left(\gamma_{n}\right)=\frac{1}{\Gamma\left(m_{n}\right)}\left(\frac{m_{n}}{\bar{\gamma}_{n}}\right)^{m_{n}} \gamma_{n}{ }^{\left(m_{n}-1\right)} \exp \left\{-\frac{m_{n}}{\bar{\gamma}_{n}} \gamma_{n}\right\}, \\
\gamma_{n}>0,
\end{array}
$$

where $m_{n}$ is the Nakagami parameter for the $n$-th sensing channel and $\bar{\gamma}_{n}=\mathrm{E}\left[\gamma_{n}\right]$ is the mean of the SNR $\gamma_{n}$.

For distinct $\bar{\gamma}_{n}$ 's, the PDF of $\gamma$ can be shown to be given in the case by

$f(\gamma)=\sum_{n=1}^{N} \sum_{j=1}^{m_{n}} \frac{A_{n j}}{\Gamma(j)}\left(\frac{m_{n}}{\bar{\gamma}_{n}}\right)^{m_{n}} \gamma^{j-1} \exp \left\{-\frac{m_{n}}{\bar{\gamma}_{n}} \gamma\right\}, \gamma>0$,

where $A_{n j}$ is determined by

$A_{n j}=\left.\frac{(-1)^{m_{n}-j}}{\left(m_{n}-j\right) !} \frac{d^{\left(m_{n}-j\right)}}{d s^{\left(m_{n}-j\right)}}\left\{M_{\gamma}(s)\left(1-\frac{\bar{\gamma}_{n}}{m_{n}} s\right)^{m_{n}}\right\}\right|_{s=\frac{m_{n}}{\bar{\gamma}_{n}}}$

with $M_{\gamma}(\cdot)$ given in the case by

$$
M_{\gamma}(s)=\left\{\prod_{n=1}^{N}\left(1-\frac{\bar{\gamma}_{n}}{m_{n}} s\right)^{-m_{n}}\right\} .
$$

Inserting (6) in (5), we get the average detection probability with the help of Eq.(29) in [9] as

$$
\bar{P}_{d \_N a k}=\sum_{n=1}^{N} \sum_{j=1}^{m_{n}} \frac{A_{n j}}{2^{j-1} \Gamma(j)}\left(\frac{m_{n}}{\bar{\gamma}_{n}}\right)^{m_{n}} G_{N u},
$$

where $G_{N u}$ can be determined recursively as

$$
\begin{aligned}
& G_{M}=G_{M-1}+ \\
& \quad \frac{\Gamma(j) \lambda^{M-1} e^{-\frac{\lambda}{2}}}{2^{M-j}(M-1) !}\left(1+\frac{m_{i}}{\bar{\gamma}_{n}}\right)^{-j}{ }_{1} F_{1}\left(j ; M ; \frac{\lambda}{2} \frac{\bar{\gamma}_{n}}{\bar{\gamma}_{n}+m_{n}}\right)
\end{aligned}
$$

with the initial $G_{1}$ given by

$$
G_{1}=\int_{0}^{\infty} x^{2 j-1} \exp \left\{-\frac{m_{n}}{2 \bar{\gamma}_{n}} x^{2}\right\} Q(x, \sqrt{\lambda}) d x,
$$

and ${ }_{1} F_{1}(\cdot ; \cdot ; \cdot)$ is the confluent hypergeometric function and $Q(\cdot, \cdot)$ is the first-order Marcum Q-function. $G_{1}$ can be written in a closed form with the help of Eq.(25) in [9] as:

$$
\begin{aligned}
G_{1} & =\frac{2^{j-1} \Gamma(j) \bar{\gamma}_{n}^{j+1}}{\left(m_{n}+\bar{\gamma}_{n}\right) m_{n}^{j}} \exp \left\{-\frac{\lambda}{2} \frac{m_{n}}{m_{n}+\bar{\gamma}_{n}}\right\} \\
& {\left[\sum_{k=0}^{j-2}\left(\frac{m_{n}}{m_{n}+\bar{\gamma}_{n}}\right)^{k} L_{k}\left(-\frac{\lambda}{2} \frac{\bar{\gamma}_{n}}{m_{n}+\bar{\gamma}_{n}}\right)+\right.} \\
& \left.\left(1+\frac{m_{n}}{\bar{\gamma}_{n}}\right)\left(\frac{m_{n}}{m_{n}+\bar{\gamma}_{n}}\right)^{j-1} L_{j-1}\left(-\frac{\lambda}{2} \frac{\bar{\gamma}_{n}}{m_{n}+\bar{\gamma}_{n}}\right)\right],
\end{aligned}
$$

where $L_{k}(\cdot)$ is the Laguerre polynomials with degree $k$. 
As a special case, the average detection probability for Rayleigh fading channels is given by making all $m_{n}=1$

$$
\begin{aligned}
\bar{P}_{d_{-} R a y} & =\sum_{n=1}^{N} A_{n}\left\{\frac{\Gamma\left(N u-1, \frac{\lambda}{2}\right)}{\Gamma(N u-1)}+\mathrm{e}^{-\frac{\lambda}{2\left(1+\bar{\gamma}_{n}\right)}}\right. \\
P & \left.\left(N u-1, \frac{\lambda}{2} \frac{\bar{\gamma}_{n}}{\left(1+\bar{\gamma}_{n}\right)}\right)\left(1+\frac{1}{\bar{\gamma}_{n}}\right)^{N u-1}\right\}
\end{aligned}
$$

where $P(M, x)=\gamma(M, x) / \Gamma(M)$ with $\gamma(M, x)=\Gamma(M)-$ $\Gamma(M, x)$ as the lower incomplete gamma function, and $A_{n}$ is determined by

$$
A_{n}=\left.M_{\gamma}(s)\left(1-\bar{\gamma}_{n} s\right)\right|_{s=1 / \bar{\gamma}_{n}}
$$

where $M_{\gamma}(s)=\left\{\prod_{n=1}^{N}\left(1-\bar{\gamma}_{n} s\right)\right\}^{-1}$.

\section{Numerical Results}

Performance of the network is often described through its receive operating characteristic (ROC) curves $\left(P_{d}\right.$ versus $\left.P_{f}\right)$ or complementary ROC curves $\left(P_{m}=1-P_{d}\right.$ versus $\left.P_{f}\right)$ for different situations of interests. In the following figures, cooperating secondary users are assumed to be uniformily distributed in a cell of radius $R$ centered around the primary user, and in this case, the average SNR $\bar{\gamma}$ for each sensing channel is assumed to be log-normal distributed with a shadow standard deviation of $\theta \mathrm{dB}$ (selected as $3 \mathrm{~dB}$ in the following simulation results), an average $\mathrm{SNR} \bar{\gamma}_{R}$ at distance $R$, and a mean value following an exponentially decreasing path loss model with an exponent $\alpha$. In this case, the PDF of average SNR $\bar{\gamma}$ is given by [10], [11]

$$
f_{\bar{\gamma}}(\bar{\gamma})=\frac{2}{c} \exp \left\{\frac{2 \theta^{2}-2 c\left(\bar{\gamma}-\bar{\gamma}_{R}\right)}{c^{2}}\right\} Q\left(\frac{2 \theta^{2}-c\left(\bar{\gamma}-\bar{\gamma}_{R}\right)}{c \cdot \theta}\right) \text {, }
$$

where $c=10 \alpha \log (e)$ is the exponential path loss parameter (with $\alpha=3$ in the following simulation results), $Q(\cdot)$ is the $Q$ function defined as the tail probability of the standard normal distribution, i.e. $Q(x)=\frac{1}{\sqrt{2 \pi}} \int_{x}^{\infty} \exp \left(-\frac{u^{2}}{2}\right) d u$.

Ignoring the fading and noise of the reporting channel, we examine the performance of energy fusion for i.n.i.d Nakagami fading channels. In Fig.1 and Fig.2, we generate the complementary ROC curves for Rayleigh fading and Nakagami fading for different values of $\bar{\gamma}_{R}$ using Neyman-Pearson Criterion. We notice that there is a great improvement in the performance with several dB's with the increase in $\bar{\gamma}_{R}$. For example, when $\bar{P}_{f}$ is set to $1 \%, \bar{P}_{m}$ decreases about 100 times as $\bar{\gamma}_{R}$ increases from $3 \mathrm{~dB}$ to $9 \mathrm{~dB}$ in Rayleigh fading conditions.

Fig.3 and Fig.4 compare the complementary ROC curves for Rayleigh and Nakagami fading conditions for different numbers $N$ of cooperative secondary users, respectively. Clearly the higher is the number of cooperative secondary users, the better is the network performance network. For example, we can see from these figures that when $\bar{P}_{f}$ is set to be $0.1 \%$, $\bar{P}_{m}$ vanishes to $10^{-12}$ in Nakagami fading channels with just 10 cooperative secondary users.

Fig.5 shows the effect of the Nakagami parameter $m$ on the complementary ROC curves for Nakagami fading channels.
There is an improvement of roughly one order of magnitude for the miss-detection probability from $m=1$ to $m=2$. This improvement diminishes when $m$ is reduced by half and increases when $m$ doubled.

\section{Conclusions}

This paper analyzed the performance of cooperative spectrum sensing over i.n.i.d Nakagami fading channels. With the scheme of energy fusion, closed form of false-alarm probability and detection probability were derived. It was shown by selected numerical results that cooperative spectrum sensing still works considerably well over i.n.i.d environment.

\section{REFERENCES}

[1] J. Mitola and G. Maguire, "Cognitive radio: Making software radios more personal," IEEE Personal Communications, vol. 6, no. 4, pp. 13$18,1999$.

[2] I. Akyildiz, W. Lee, M. Vuran, and S. Mohanty, "NeXt generation/dynamic spectrum access/cognitive radio wireless networks: A survey," Computer Networks, vol. 50, no. 13, pp. 2127-2159, 2006.

[3] C. Sun, W. Zhang, and K. Letaief, "Cluster-based cooperative spectrum sensing in cognitive radio systems," in Proc. of the IEEE International Conference on Communications (ICC'07), pp. 2511-2515, Glasgow, Scotland, June, 2007.

[4] W. Zhang, R. Mallik, and K. Letaief, "Cooperative spectrum sensing optimization in cognitive radio networks," in Proc. of the IEEE International Conference on Communications (ICC'08), pp. 3411-3415, Beijing, China, May, 2008.

[5] F. Digham, M.-S. Alouini, and M. Simon, "On the energy detection of unknown signals over fading channels," IEEE Transactions on Communications, vol. 55, no. 1, pp. 21-24, 2007.

[6] M. Nakagami, "The m-distribution- A general formula of intensity distribution of rapid fading," in Statistical methods in radio wave propagation (W. G. Hoffman, ed.), pp. 3-36, Pergamon Press, Oxford, England, 1960.

[7] H. Suzuki, "A statistical model for urban multipath propagation," IEEE Trans. Commun, vol. 25, no. 7, pp. 673-680, 1977.

[8] M. H. A. U. Sheikh and M. Abdi, "Indoor mobile radio channel at $946 \mathrm{MHz}$ : Measurements and modeling," in Proc. IEEE Vehicular Technology Conference (VTC'93), pp. 73-76, Secaucus, NJ, USA, May, 1993.

[9] A. Nuttall, "Some Integrals Involving the $Q_{M}$-Function," Naval Underwater Systems Center (NUSC) Technical Report, May 1974.

[10] L. Yang, M. Kang, and M.-S. Alouini, "On the capacity-fairness tradeoff in multiuser diversity systems," IEEE Transactions on Vehicular Technology, vol. 56, no. 4 Part 1, pp. 1901-1907, 2007.

[11] M. Yacoub, Foundations of Mobile Radio Engineering. CRC Press, Inc. Boca Raton, FL, USA, 1993.

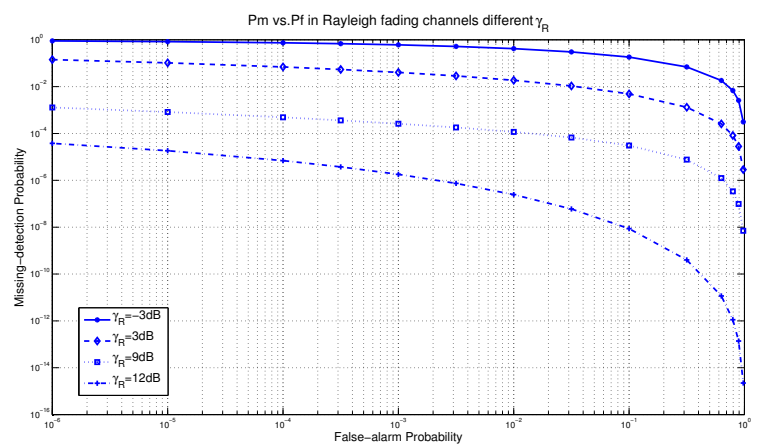

Fig. 1. Complementary ROC curves for Rayleigh fading channels for different $\bar{\gamma}_{R}$ values $(N=5$ and $u=5)$. 


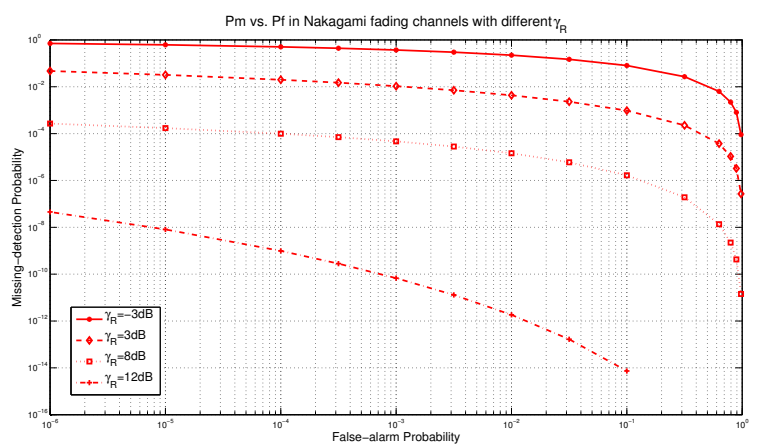

Fig. 2. Complementary ROC curves for Nakagami fading channels for different $\bar{\gamma}_{R}$ values with Nakagami parameter $m=3(N=5$ and $u=5)$

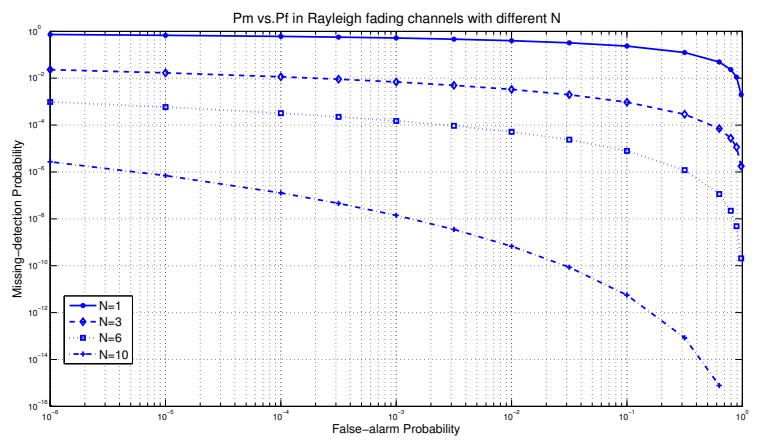

Fig. 3. Complementary ROC curves for Rayleigh fading channels for different $N$ values $\left(\bar{\gamma}_{R}=8 \mathrm{~dB}\right.$ and $\left.u=5\right)$.

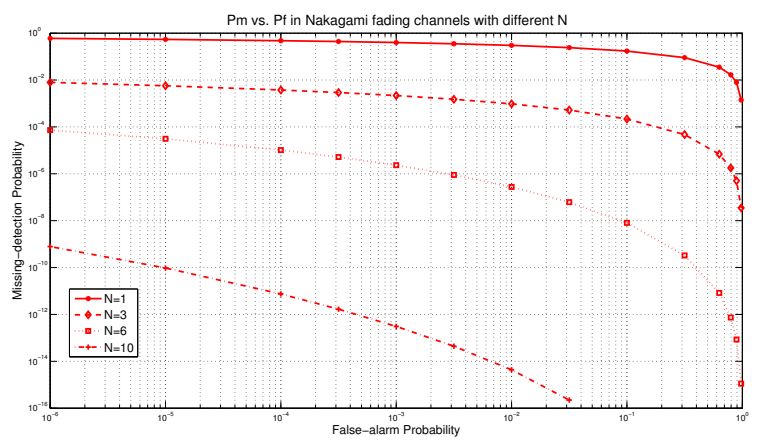

Fig. 4. Complementary ROC curves for Nakagami fading channels with different values of $N$ for a Nakagami parameter $m=3\left(\bar{\gamma}_{R}=8 \mathrm{~dB}\right.$ and $u=5)$.

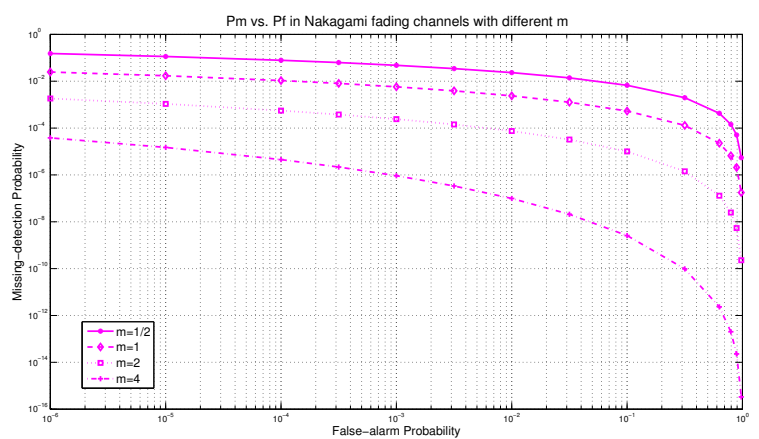

Fig. 5. Complementary ROC curves for Nakagami fading channels with different values of $m\left(\bar{\gamma}_{R}=8 \mathrm{~dB}, N=5\right.$, and $\left.u=5\right)$. 